\title{
Neighbourhood $V_{4}$-Magic Labeling of Some Splitting Graphs
}

\author{
Vineesh K.P. ${ }^{1}$ and Anil Kumar V. ${ }^{2}$ \\ ${ }^{1}$ Department of Mathematics,Sree Narayana Guru College, Chelannur, Kozhikode, \\ Kerala,India 673616 \\ ${ }^{2}$ Department of Mathematics, University of Calicut, Malappuram, Kerala, India \\ 673 635, email:kpvineeshmaths@gmail.com ${ }^{1}$, anil@uoc.ac.in ${ }^{2}$
}

\begin{abstract}
The Klein 4-group,denoted by $V_{4}$ is an abelian group of order 4 . It has elements $V_{4}=\{0, a, b, c\}$, with $a+a=b+b=$ $c+c=0$ and $a+b=c, b+c=a, c+a=$ b. A graph $G(V(G), E(G)$ is said to be neighbourhood $V_{4}$-magic if there exists a labeling $f: V(G) \rightarrow V_{4} \backslash\{0\}$ such that the induced mapping $N_{f}^{+}: V(G) \rightarrow V_{4}$ defined by $N_{f}^{+}(v)=\sum_{u \in N(v)} f(u)$ is a constant map. If this constant is $p(p \neq 0)$, we say that $f$ is a $p$ - neighbourhood $V_{4}$-magic labeling of $G$ and $G$ a $p$ - neighbourhood $V_{4}$ - magic graph. If this constant is zero, we say that $f$ is a 0 -neighghbourhood $V_{4}$-magic labeling of $G$ and $G$ a 0 -neighbourhood $V_{4}$ - magic graph. In this paper, we discuss neighbourhood $V_{4}$ - magic labeling of some splitting graphs.

Key words: Klein-4-group, Splitting graphs, $a$-neighbourhood and 0 -neighbourhood $V_{4}$-magic graphs. AMS classification: 05C78, 05C25.
\end{abstract}

\section{Introduction}

Throughout this paper we consider simple, finite, connected and undirected graphs. For standard terminology and notation we follow [1] and [6]. For a detailed survey on graph labeling we refer [2]. The $V_{4}$-magic graphs were intro- duced by S. M. Lee et al. in 2002 [3]. We say that, a graph $G=(V(G), E(G))$, with vertex set $V(G)$ and edge set $E(G)$ is Neighbourhood $V_{4}$-magic if there exists a labeling $f: V(G) \rightarrow$ $V_{4} \backslash\{0\}$ such that the induced mapping $N_{f}^{+}$: $V(G) \rightarrow V_{4}$ defined by $N_{f}^{+}(v)=\sum_{u \in N(v)} f(u)$ is a constant map. If this constant is $p$, where $p$ is any non zero element in $V_{4}$, then we say that $f$ is a $p$-neighbourhood $V_{4}$-magic labeling of $G$ and $G$ is said to be a $p$-neighbourhood $V_{4}$-magic graph. If this constant is 0 , then we say that $f$ is a 0 - neighbourhood $V_{4}$ magic labeling of $G$ and $G$ is said to be a 0 -neighbourhood $V_{4}$-magic graph. We divide the class of neighbourhood $V_{4}$-magic graphs into the following three categories:

(i) $\Omega_{a}:=$ the class of all $a-$ neighbourhood $V_{4}$-magic graphs,

(ii) $\Omega_{0}:=$ the class of all $0-$ neighbourhood $V_{4}$-magic graphs, and

(iii) $\Omega_{a, 0}:=\Omega_{a} \cap \Omega_{0}$.

The Splitting graph $S(G)$ of a connected graph $G$ is obtained by adding to each vertex $u$ in $G$, a new vertex $u^{\prime}$ such that $u^{\prime}$ is adjacent to the neighbours of $u$ in $G$. The Bistar $B_{m, n}$ is the graph obtained by joining the central vertex $K_{1, m}$ and $K_{1, n}$ by an edge[2].The friendship graph or the Dutch windmill graph, denoted by $F_{m}$ (or $D_{3}^{(m)}$ ) is obtained by taking $m$ copies 
of $C_{3}$ with one vertex in common[5]. The Book graph $B_{n}$ is the graph $S_{n} \square P_{2}$, where $S_{n}$ is the star with $n+1$ vertices and $P_{2}$ is the path on 2 vertices[7]. A quadrilateral snake $Q S_{n}$ is obtained from a path $v_{1} v_{2} v_{3} \ldots v_{n}$ by joining each pair $v_{i}, v_{i+1}$ to the new vertices $u_{i}, w_{i}$ respectively and then joining $u_{i}$ and $w_{i}$ by an edge[4]. This paper investigate neighbourhood $V_{4}$ - magic labeling of splitting graphs of $C_{n}, P_{n}, B_{m, n}, K_{1, n}, K_{m, n}, F_{m}, Q S_{n}$ and $B_{n}$.

\section{Main Results}

Theorem 2.1. The graph $S\left(C_{n}\right) \in \Omega_{a}$ if and only if $n \equiv 0(\bmod 4)$.

Proof. Consider the splitting graph $S\left(C_{n}\right)$, let $u_{1}, u_{2}, u_{3}, \ldots, u_{n}$ be the vertices of $C_{n}$ and let $u_{1}^{\prime}, u_{2}^{\prime}, u_{3}^{\prime}, \ldots, u_{n}^{\prime}$ be the new vertices in $S\left(C_{n}\right)$. Assume that $n \not \equiv 0(\bmod 4)$. Then either $n \equiv 1(\bmod 4)$ or $n \equiv 2(\bmod 4)$ or $n \equiv 3(\bmod 4)$. We show that in each these cases $S\left(C_{n}\right) \notin \Omega_{a}$.

Case 1: $n \equiv 1(\bmod 4)$

In this case $n=4 k+1$ for some $k \in \mathbb{N}$. Then $V\left(S\left(C_{n}\right)\right)=\left\{u_{i}, u_{i}^{\prime}: 1 \leq i \leq 4 k+1\right\}$. If possible let $S\left(C_{n}\right) \in \Omega_{a}$ with a labeling $f$. Then $N_{f}^{+}\left(u_{2}^{\prime}\right)=a$ implies that $f\left(u_{1}\right)+$ $f\left(u_{3}\right)=a$, which implies that either $f\left(u_{1}\right)=b$ or $f\left(u_{1}\right)=c$. Without loss of generality assume that $f\left(u_{1}\right)=b$. Then $f\left(u_{3}\right)=c, f\left(u_{5}\right)=$ $b, f\left(u_{7}\right)=c, f\left(u_{9}\right)=b, f\left(u_{11}\right)=c, f\left(u_{13}\right)=b$. Proceeding like this, we get $f\left(u_{4 k+1}\right)=b$. Also $N_{f}^{+}\left(u_{1}^{\prime}\right)=a$ and $f\left(u_{4 k+1}\right)=b$ implies that $f\left(u_{2}\right)=c, f\left(u_{4}\right)=b, f\left(u_{6}\right)=c, f\left(u_{8}\right)=$ $b, f\left(u_{10}\right)=c, f\left(u_{12}\right)=b$. Proceeding like this we get $f\left(u_{4 k}\right)=b$. Therefore, $N_{f}^{+}\left(u_{4 k+1}^{\prime}\right)=$ $b+b=0$, a contradiction. Thus if $n \equiv 1(\bmod 4)$, we have $S\left(C_{n}\right) \notin \Omega_{a}$.

Case 2: $n \equiv 2(\bmod 4)$

In this case $n=4 k+2$ for some $k \in \mathbb{N}$. Then $V\left(S\left(C_{n}\right)\right)=\left\{u_{i}, u_{i}^{\prime}: 1 \leq i \leq 4 k+2\right\}$. If possible let $S\left(C_{n}\right) \in \Omega_{a}$ with a labeling $f$. Then $N_{f}^{+}\left(u_{2}^{\prime}\right)=a$ implies that $f\left(u_{1}\right)+$ $f\left(u_{3}\right)=a$, which implies that either $f\left(u_{1}\right)=b$ or $f\left(u_{1}\right)=c$. Without loss of generality as- sume that $f\left(u_{1}\right)=b$. Then $f\left(u_{3}\right)=c, f\left(u_{5}\right)=$ $b, f\left(u_{7}\right)=c, f\left(u_{9}\right)=b, f\left(u_{11}\right)=c, f\left(u_{13}\right)=b$. Proceeding like this, we get $f\left(u_{4 k+1}\right)=b$. Now $N_{f}^{+}\left(u_{4 k+2}^{\prime}\right)=f\left(u_{1}\right)+f\left(u_{4 k+1}\right)=b+b=0, \mathrm{a}$ contradiction. Thus if $n \equiv 2(\bmod 4)$, we have $S\left(C_{n}\right) \notin \Omega_{a}$.

Case 3: $n \equiv 3(\bmod 4)$

In this case $n=4 k+3$ for some $k \in \mathbb{N}$. Then $V\left(S\left(C_{n}\right)\right)=\left\{u_{i}, u_{i}^{\prime}: 1 \leq i \leq 4 k+3\right\}$. If possible let $S\left(C_{n}\right) \in \Omega_{a}$ with a labeling $f$. Then $N_{f}^{+}\left(u_{2}^{\prime}\right)=a$ implies that $f\left(u_{1}\right)+$ $f\left(u_{3}\right)=a$, which implies that either $f\left(u_{1}\right)=b$ or $f\left(u_{1}\right)=c$. Without loss of generality assume that $f\left(u_{1}\right)=b$. Then $f\left(u_{3}\right)=c, f\left(u_{5}\right)=$ $b, f\left(u_{7}\right)=c, f\left(u_{9}\right)=b, f\left(u_{11}\right)=c, f\left(u_{13}\right)=b$. Proceeding like this, we get $f\left(u_{4 k+3}\right)=c$. Now $N_{f}^{+}\left(u_{1}^{\prime}\right)=a$ implies that $f\left(u_{2}\right)=b, f\left(u_{4}\right)=$ $c, f\left(u_{6}\right)=b, f\left(u_{8}\right)=c, f\left(u_{10}\right)=b, f\left(u_{12}\right)=c$. Proceeding like this, we get $f\left(u_{4 k+2}\right)=b$. Therefore $N_{f}^{+}\left(u_{4 k+3}^{\prime}\right)=f\left(u_{1}\right)+f\left(u_{4 k+2}\right)=b+$ $b=0$, a contradiction. Thus if $n \equiv 3(\bmod 4)$, we also have $S\left(C_{n}\right) \notin \Omega_{a}$. Hence if $n \not \equiv 0(\bmod 4)$, $S\left(C_{n}\right) \notin \Omega_{a}$. Conversely if $n \equiv 0(\bmod 4)$,

Define $f: V\left(S\left(C_{n}\right)\right) \rightarrow V_{4} \backslash\{0\}$ as:

$$
\begin{aligned}
& f\left(u_{i}\right)=\left\{\begin{array}{lll}
b & \text { if } & i \equiv 1,2(\bmod 4) \\
c & \text { if } & i \equiv 0,3(\bmod 4)
\end{array}\right. \\
& f\left(u_{i}^{\prime}\right)=a \quad \text { for } \quad 1 \leq i \leq n .
\end{aligned}
$$

Then, $f$ is a $a$-neighbourhood $V_{4}$-magic labeling for $S\left(C_{n}\right)$. This completes the proof of the theorem.

Theorem 2.2. $S\left(C_{n}\right) \in \Omega_{0}$ for all $n \geq 3$.

Proof. The degree of each vertex in $S\left(C_{n}\right)$ is either 2 or 4 . By labeling all the vertices by $a$, we get $N_{f}^{+}(u)=0$ for all $u \in V\left(S\left(C_{n}\right)\right)$.

Corollary 2.3. $S\left(C_{n}\right) \in \Omega_{a, 0}$ if and only if $n \equiv 0(\bmod 4)$.

Proof. Proof is obviously follows from theorem 2.1 and theorem 2.2 .

Theorem 2.4. The graph $S\left(P_{n}\right) \notin \Omega_{0}$ for all $n \geq 2$. 
Proof. Proof is obvious due to the presence of pendant vertices in $S\left(P_{n}\right)$.

Theorem 2.5. $S\left(P_{n}\right) \notin \Omega_{a}$ for $n \geq 2$.

Proof. Consider the splitting graph $S\left(P_{n}\right)$, let $u_{1}, u_{2}, u_{3}, \ldots, u_{n}$ be the vertices of $P_{n}$ and let $u_{1}^{\prime}, u_{2}^{\prime}, u_{3}^{\prime}, \ldots, u_{n}^{\prime}$ be the new vertices in $S\left(P_{n}\right)$. Suppose that $S\left(P_{n}\right) \in \Omega_{a}$ for some $n \geq 2$ with a labeling $f$. Then $N_{f}^{+}\left(u_{1}^{\prime}\right)=a$ implies that $f\left(u_{2}\right)=a$. Also $N_{f}^{+}\left(u_{1}^{\prime}\right)=a$ gives $f\left(u_{2}\right)+f\left(u_{2}^{\prime}\right)=a$, which implies that $f\left(u_{2}^{\prime}\right)=0$, a contradiction. This completes the proof of the theorem.

Corollary 2.6. $S\left(P_{n}\right) \notin \Omega_{a, 0}$ for $n \geq 2$.

Proof. Proof directly follows from theorems 2.4 and 2.5.

Theorem 2.7. $S\left(B_{m, n}\right) \notin \Omega_{a}$ for all $m>1$ and $n>1$.

Proof. Consider the bistar $B_{m, n}$ with vertex set $V=\left\{u, v, u_{i}, v_{j}: 1 \leq i \leq m\right.$ and $\left.1 \leq j \leq n\right\}$ where $u_{i}(1 \leq i \leq m)$ and $v_{j}(1 \leq j \leq n)$ are pendant vertices adjacent to $u$ and $v$ respectively. Let $V^{\prime}=\left\{u^{\prime}, v^{\prime}, u_{i}^{\prime}, v_{j}^{\prime}: 1 \leq i \leq\right.$ $m$ and $1 \leq j \leq n\}$ be the corresponding set of new vertices in $S\left(B_{m, n}\right)$. Then $V\left(S\left(B_{m, n}\right)\right)=$ $V \cup V^{\prime}$. Suppose that $S\left(B_{m, n}\right) \in \Omega_{a}$ for some $m>1$ and $n>1$ with a labeling $f$. Then $N_{f}^{+}\left(v_{1}^{\prime}\right)=a$ implies that $f(v)=a$. Now $N_{f}^{+}\left(v_{1}\right)=a$ gives $f(v)+f\left(v^{\prime}\right)=a$, which implies that $f\left(v^{\prime}\right)=0$, a contradiction. This completes the proof of the theorem.

Theorem 2.8. $S\left(B_{m, n}\right) \notin \Omega_{0}$ for all $m>1$ and $n>1$.

Proof. Proof is obvious, since $S\left(B_{m, n}\right)$ has pendant vertices.

Corollary 2.9. $S\left(B_{m, n}\right) \notin \Omega_{a, 0}$ for all $m>1$ and $n>1$.

Proof. Proof directly follows from theorems 2.7 and 2.8 .

Theorem 2.10. $S\left(K_{1, n}\right) \notin \Omega_{a}$ for all $n \in \mathbb{N}$.
Proof. Consider $K_{1, n}$ with vertex set $V=$ $\left\{u, u_{i}: 1 \leq i \leq n\right\}$ and let $V^{\prime}=\left\{u^{\prime}, u_{i}^{\prime}\right.$ : $1 \leq i \leq n\}$ be the corresponding set of new vertices in $S\left(K_{1, n}\right)$. Assume that $S\left(K_{1, n}\right) \in$ $\Omega_{a}$ for some $n \in \mathbb{N}$ with a labeling $f$. Now $N_{f}^{+}\left(u_{1}^{\prime}\right)=a$ gives $f(u)=a$. Also $N_{f}^{+}\left(u_{1}\right)=a$ implies that $f(u)+f\left(u^{\prime}\right)=a$, which implies that $f\left(u^{\prime}\right)=0$, a contradiction. Hence the proof.

Theorem 2.11. $S\left(K_{1, n}\right) \notin \Omega_{0}$ for all $n \in \mathbb{N}$.

Proof. Proof is obvious due to the presence of pendant vertices in $S\left(K_{1, n}\right)$.

Corollary 2.12. $S\left(K_{1, n}\right) \notin \Omega_{a, 0}$ for all $n \in$ $\mathbb{N}$.

Proof. Proof directly follows from theorems 2.10 and 2.11.

Theorem 2.13. $S\left(K_{m, n}\right) \in \Omega_{a}$ for all $m>1$ and $n>1$.

Proof. Consider $K_{m, n}$ with $m>1$ and $n>$ 1. Let $X=\left\{u_{1}, u_{2}, u_{3}, \ldots, u_{m}\right\}$ and $Y=$ $\left\{v_{1}, v_{2}, v_{3}, \ldots, v_{n}\right\}$ be the bipartition of $K_{m, n}$. Also let $X^{\prime}=\left\{u_{1}^{\prime}, u_{2}^{\prime}, u_{3}^{\prime}, \ldots, u_{m}^{\prime}\right\}$ and $Y^{\prime}=$ $\left\{v_{1}^{\prime}, v_{2}^{\prime}, v_{3}^{\prime}, \ldots, v_{n}^{\prime}\right\}$ be the corresponding sets of new vertices in $S\left(K_{m, n}\right)$. Then $V\left(S\left(K_{m, n}\right)\right)=$ $X \cup Y \cup X^{\prime} \cup Y^{\prime}$. We consider the following cases:

Case 1: Both $m$ and $n$ are even.

Define $f: V\left(S\left(K_{m, n}\right)\right) \rightarrow V_{4} \backslash\{0\}$ as:

$$
\begin{aligned}
& f\left(u_{i}\right)=\left\{\begin{array}{lll}
b & \text { if } & i=1 \\
c & \text { if } & i=2 \\
a & \text { if } & i>2
\end{array}\right. \\
& f\left(v_{j}\right)=\left\{\begin{array}{lll}
b & \text { if } & j=1 \\
c & \text { if } & j=2 \\
a & \text { if } & j>2
\end{array}\right. \\
& f\left(u_{i}^{\prime}\right)=a \text { for } 1 \leq i \leq m \\
& f\left(v_{j}^{\prime}\right)=a \text { for } 1 \leq j \leq n
\end{aligned}
$$


Case 2: $m$ is even and $n$ is odd.

$$
\begin{aligned}
& f\left(u_{i}\right)=\left\{\begin{array}{lll}
b & \text { if } & i=1 \\
c & \text { if } & i=2 \\
a & \text { if } & i>2
\end{array}\right. \\
& f\left(v_{j}^{\prime}\right)=\left\{\begin{array}{lll}
b & \text { if } & j=1 \\
c & \text { if } & j=2 \\
a & \text { if } & j>2
\end{array}\right. \\
& f\left(u_{i}^{\prime}\right)=a \text { for } 1 \leq i \leq m \\
& f\left(v_{j}\right)=a \text { for } 1 \leq j \leq n
\end{aligned}
$$

Case 3: $m$ is odd and $n$ is even.

$$
\begin{aligned}
& f\left(u_{i}^{\prime}\right)=\left\{\begin{array}{lll}
b & \text { if } & i=1 \\
c & \text { if } & i=2 \\
a & \text { if } & i>2
\end{array}\right. \\
& f\left(v_{j}\right)=\left\{\begin{array}{lll}
b & \text { if } & j=1 \\
c & \text { if } & j=2 \\
a & \text { if } & j>2
\end{array}\right. \\
& f\left(u_{i}\right)=a \text { for } 1 \leq i \leq m
\end{aligned}
$$

Case 4: Both $m$ and $n$ are odd.

$$
\begin{aligned}
& f\left(u_{i}^{\prime}\right)=\left\{\begin{array}{lll}
b & \text { if } & i=1 \\
c & \text { if } & i=2 \\
a & \text { if } & i>2
\end{array}\right. \\
& f\left(v_{j}^{\prime}\right)=\left\{\begin{array}{lll}
b & \text { if } & j=1 \\
c & \text { if } & j=2 \\
a & \text { if } & j>2
\end{array}\right. \\
& f\left(u_{i}\right)=a \text { for } 1 \leq i \leq m
\end{aligned}
$$

In each of the above cases, $f$ is a $a$ neighbourhood $V_{4}$-magic labeng of $S\left(K_{m, n}\right)$. This completes the proof of the theorem.

Theorem 2.14. $S\left(K_{m, n}\right) \in \Omega_{0}$ for all $m>1$ and $n>1$.
Proof. Consider $K_{m, n}$ with $m>1$ and $n>$ 1. Let $X=\left\{u_{1}, u_{2}, u_{3}, \ldots, u_{m}\right\}$ and $Y=$ $\left\{v_{1}, v_{2}, v_{3}, \ldots, v_{n}\right\}$ be the bipartition of $K_{m, n}$. Also let $X^{\prime}=\left\{u_{1}^{\prime}, u_{2}^{\prime}, u_{3}^{\prime}, \ldots, u_{m}^{\prime}\right\}$ and $Y^{\prime}=$ $\left\{v_{1}^{\prime}, v_{2}^{\prime}, v_{3}^{\prime}, \ldots, v_{n}^{\prime}\right\}$ be the corresponding sets of new vertices in $S\left(K_{m, n}\right)$. Then $V\left(S\left(K_{m, n}\right)\right)=$ $X \cup Y \cup X^{\prime} \cup Y^{\prime}$. We consider the following cases:

Case 1: Both $m$ and $n$ are even.

Define $f: V\left(S\left(K_{m, n}\right)\right) \rightarrow V_{4} \backslash\{0\}$ as:

$$
\begin{aligned}
& f\left(u_{i}\right)=f\left(u_{i}^{\prime}\right)=a \quad \text { if } \quad i=1,2,3, \ldots, m \\
& f\left(v_{j}\right)=f\left(v_{j}^{\prime}\right)=a \quad \text { if } \quad j=1,2,3, \ldots, n
\end{aligned}
$$

Case 2: $m$ is even and $n$ is odd.

$$
\begin{aligned}
& f\left(v_{j}\right)=\left\{\begin{array}{lll}
b & \text { if } & j=1 \\
c & \text { if } & j=2 \\
a & \text { if } & j>2
\end{array}\right. \\
& f\left(v_{j}^{\prime}\right)=\left\{\begin{array}{lll}
b & \text { if } & j=1 \\
c & \text { if } & j=2 \\
a & \text { if } & j>2
\end{array}\right. \\
& f\left(u_{i}\right)=a \text { for } 1 \leq i \leq m \\
& f\left(u_{i}^{\prime}\right)=a \text { for } 1 \leq i \leq m
\end{aligned}
$$

Case 3: $m$ is odd and $n$ is even.

$$
\begin{aligned}
& f\left(u_{i}\right)=\left\{\begin{array}{lll}
b & \text { if } & i=1 \\
c & \text { if } & i=2 \\
a & \text { if } & i>2
\end{array}\right. \\
& f\left(u_{i}^{\prime}\right)=\left\{\begin{array}{lll}
b & \text { if } & i=1 \\
c & \text { if } & i=2 \\
a & \text { if } & i>2
\end{array}\right. \\
& f\left(v_{j}\right)=a \text { for } 1 \leq j \leq n \\
& f\left(v_{j}^{\prime}\right)=a \text { for } 1 \leq j \leq n
\end{aligned}
$$

Case 4: Both $m$ and $n$ are odd.

$$
f\left(u_{i}\right)=\left\{\begin{array}{lll}
b & \text { if } & i=1 \\
c & \text { if } & i=2 \\
a & \text { if } & i>2
\end{array}\right.
$$




$$
\begin{array}{r}
f\left(u_{i}^{\prime}\right)=\left\{\begin{array}{lll}
b & \text { if } & i=1 \\
c & \text { if } & i=2 \\
a & \text { if } & i>2
\end{array}\right. \\
f\left(v_{j}\right)=\left\{\begin{array}{lll}
b & \text { if } & j=1 \\
c & \text { if } & j=2 \\
a & \text { if } & j>2
\end{array}\right. \\
f\left(v_{j}^{\prime}\right)=\left\{\begin{array}{lll}
b & \text { if } & j=1 \\
c & \text { if } & j=2 \\
a & \text { if } & j>2
\end{array}\right.
\end{array}
$$

In each of the above cases, $f$ is a 0 neighbourhood $V_{4}$-magic labeng of $S\left(K_{m, n}\right)$. This completes the proof of the theorem.

Corollary 2.15. $S\left(K_{m, n}\right) \in \Omega_{a, 0}$ for all $m>$ 1 and $n>1$.

Proof. Proof directly follows from theorems 2.13 and 2.14.

Theorem 2.16. $S\left(F_{m}\right) \in \Omega_{0}$ for all $m \in \mathbb{N}$.

Proof. If we label all the vertices of $S\left(F_{m}\right)$ by $a$, we get $S\left(F_{m}\right) \in \Omega_{0}$.

Theorem 2.17. $S\left(F_{m}\right) \notin \Omega_{a}$ for all $m \in \mathbb{N}$.

Proof. Consider the friendship graph $F_{m}$. Let the vertices of $i^{t h}$ copy of $C_{3}$ in $F_{m}$ be $w, u_{i}$ and $v_{i}$ where $w$ is the common vertex of the triangles and let $\left\{w^{\prime}, u_{i}^{\prime}, v_{i}^{\prime}: 1 \leq i \leq m\right\}$ be the corresponding set of vertices in $S\left(\bar{F}_{m}\right)$. Assume that $S\left(F_{m}\right) \in \Omega_{a}$ for some $m \in \mathbb{N}$ with a labeling $f$. Since $N_{f}^{+}\left(u_{1}^{\prime}\right)=a$, either $f(w)=b$ or $f(w)=c$. Without loss of generality assume that $f(w)=b$. If $f(w)=b, f\left(u_{i}\right)=f\left(v_{i}\right)=c$ for all $1 \leq i \leq m$. Therefore, $N_{f}^{+}\left(w^{\prime}\right)=2 m c=$ 0 , a contradiction. Hence $S\left(F_{m}\right) \notin \Omega_{a}$ for all $m \in \mathbb{N}$.

Corollary 2.18. $S\left(F_{m}\right) \notin \Omega_{a, 0}$ for all $m \in \mathbb{N}$.

Proof. Proof directly follows from theorems 2.16 and 2.17.

Theorem 2.19. $S\left(Q S_{n}\right) \in \Omega_{0}$ for all $n \in \mathbb{N}$.
Proof. Label all the vertices of $S\left(Q S_{n}\right)$ by $a$, we get $S\left(Q S_{n}\right) \in \Omega_{0}$.

Theorem 2.20. $S\left(Q S_{n}\right) \notin \Omega_{a}$ for all $n>2$.

Proof. Let $Q S_{n}$ be the quadrilateral snake obtained from the path $v_{1} v_{2} v_{3} \ldots v_{n}$ by joining each pair $v_{i}, v_{i+1}$ to the new vertices $u_{i}, w_{i}$ respectively and then joining $u_{i}$ and $w_{i}$ by an edge. Now consider $S\left(Q S_{n}\right)$. Let $v_{i}^{\prime}, u_{i}^{\prime}, w_{i}^{\prime}$ be the new vertices corresponding to $v_{i}, u_{i}, w_{i}$. Suppose $S\left(Q S_{n}\right) \in \Omega_{a}$ for some $n>2$ with a labeling $f$. Then, $N_{f}^{+}\left(u_{1}^{\prime}\right)=a$ gives $f\left(v_{1}\right)+f\left(w_{1}\right)=a$. Also $N_{f}^{+}\left(w_{2}^{\prime}\right)=a$ implies that $f\left(u_{2}\right)+f\left(v_{3}\right)=a$, Therefore, $N_{f}^{+}\left(v_{2}^{\prime}\right)=$ $f\left(v_{1}\right)+f\left(w_{1}\right)+f\left(u_{2}\right)+f\left(v_{3}\right)=0$, a contradiction. Hence, $S\left(Q S_{n}\right) \notin \Omega_{a}$ for all $n>2$.

Corollary 2.21. $S\left(Q S_{n}\right) \notin \Omega_{a, 0}$ for all $n>2$.

Proof. Proof directly follows from theorems 2.19 and 2.20 .

Theorem 2.22. $S\left(B_{n}\right) \in \Omega_{a}$ if and only if $n$ is odd.

Proof.

Consider $B_{n}$ with vertex set $\left\{u, v, u_{i}, v_{i}: 1 \leq\right.$ $i \leq n\}$ and edge set $\left\{u v, u u_{i}, v v_{i}, u_{i} v_{i}: 1 \leq\right.$ $i \leq n\}$. Let $\left\{u^{\prime}, v^{\prime}, u_{i}^{\prime}, v_{i}^{\prime}: 1 \leq i \leq n\right\}$ be the set of new vertices in $S\left(B_{n}\right)$. Assume that $S\left(B_{n}\right) \in \Omega_{a}$ for some $n \in \mathbb{N}$ with a labeling $f$. Since $N_{f}^{+}\left(u_{1}^{\prime}\right)=a$, we have $f(u)=b$ or $f(u)=c$. Without loss of generality we assume that $f(u)=b$. Then $f\left(v_{i}\right)=c$ for all $i=1,2,3, \ldots, n$. Now $N_{f}^{+}\left(v^{\prime}\right)=a$ implies that $f(u)+\sum_{i=1}^{n} f\left(v_{i}\right)=b+n c=a$. Hence $n$ is odd. Conversely assume that $n$ is odd. Define a labeling $f: V\left(S\left(B_{n}\right)\right) \rightarrow V_{4} \backslash\{0\}$ as:

$$
\begin{array}{lll}
f(u)=f\left(u_{i}\right)=b & \text { if } & i=1,2,3, \ldots, n \\
f(v)=f\left(v_{i}\right)=c & \text { if } & i=1,2,3, \ldots, n \\
\left.f\left(u^{\prime}\right)=f u_{i}^{\prime}\right)=a & \text { if } & i=1,2,3, \ldots, n \\
f(v)=f\left(v_{i}^{\prime}\right)=a & \text { if } & i=1,2,3, \ldots, n
\end{array}
$$

Then, $f$ is a $a$-neighbourhood $V_{4}$-magic labeling of $S\left(B_{n}\right)$. This completes the proof. 
Theorem 2.23. $S\left(B_{n}\right) \in \Omega_{0}$ if and only if $n$ is odd.

Proof. Consider the book graph $B_{n}$ with vertex set $\left\{u, v, u_{i}, v_{i}: 1 \leq i \leq n\right\}$ and edge set $\left\{u v, u u_{i}, v v_{i}, u_{i} v_{i}: 1 \leq i \leq n\right\}$. Let $\left\{u^{\prime}, v^{\prime}, u_{i}^{\prime}, v_{i}^{\prime}: 1 \leq i \leq n\right\}$ be the set of new vertices in $S\left(B_{n}\right)$. Assume that $S\left(B_{n}\right) \in \Omega_{0}$ for some $n \in \mathbb{N}$ with a labeling $f$. Since $N_{f}^{+}\left(u_{1}^{\prime}\right)=0$, we should have $f(u)=f\left(v_{1}\right)=a$ or $f(u)=f\left(v_{1}\right)=b$ or $f(u)=f\left(v_{1}\right)=$ $c$. Without loss of generality we assume that $f(u)=f\left(v_{1}\right)=a$. Then $f\left(v_{i}\right)=a$ for all $i=1,2,3, \ldots, n$. Now $N_{f}^{+}\left(v^{\prime}\right)=0$ implies that $f(u)+\sum_{i=1}^{n} f\left(v_{i}\right)=a+n a=0$. Hence $n$ is odd. Conversely assume that $n$ is odd. We define $f: V\left(S\left(B_{n}\right)\right) \rightarrow V_{4} \backslash\{0\}$ as: $f(w)=a$ for all $w \in V\left(S\left(B_{n}\right)\right)$. Then, $f$ is a 0 - neighbourhood $V_{4}$-magic labeling of $S\left(B_{n}\right)$.

Corollary 2.24. $S\left(B_{n}\right) \in \Omega_{a, 0}$ if and only if $n$ is odd.

Proof. Proof directly follows from theorems 2.22 and 2.23 .

This research paper investigates neighbourhood $V_{4}$ - magic labeling of splitting graphs of special graphs like $C_{n}, P_{n}, B_{m, n}, K_{1, n}, K_{m, n}$, $F_{m}, Q S_{n}$ and $B_{n}$ respectively. The splitting graph we considered was 2-level splits. The $m$ - level splitting graphs $(m>2)$ yet to be considered. Scope of this research is the investigation of neighbourhood $V_{4}$-magic labeling of $m$ - level splitting graphs for $m>2$.

\section{References}

[1] Chartrand.G,Zhang.P, Introduction to Graph Theory, McGraw-Hill, 2005.

[2] Joseph A.Gallian, $A$ dynamic survey of graph labeling, The Electronics Journal of Combinatorics, Twenty-first edition, December 21, 2018.
[3] Lee SM, Saba F, Salehi E, Sun H. On the $V_{4}-$ magic graphs, Congressus Numerantium,2002.

[4] N. B. Rathod and K. K. Kanani, $V_{4}$-Cordial Labeling of Quadrilateral Snakes, International Journal of emerging Technologies and applications in Engineering, Technology and Sciences,Jan 2016.

[5] P.T Vandana, V anil Kumar: $V_{4}$-Magic Labelings of Wheel related graphs, British journal of Mathematics and Computer science,8(3),189-219,(2015).

[6] R. Balakrishnan and K. Ranganathan, A Textbook of Graph theory, Springer, 2012.

[7] R.Sweetly and J.Paulraj Joseph,Some special $V_{4}-$ magic graphs, journal of informatics and Mathematical sciences,2,141$148,(2010)$. 